\title{
Future-oriented Generic Grid Code regarding Wind Power Plants in Europe
}

\author{
Til Kristian Vrana ${ }^{\mathrm{a}}$, Ayman Attya ${ }^{\mathrm{b}}$, Lluís Trilla ${ }^{\mathrm{c}}$ \\ ${ }^{a}$ SINTEF Energi, Norway \\ ${ }^{b}$ University of Huddersfield, UK \\ ${ }^{c}$ IREC, Catalunya, Spain
}

\begin{abstract}
The assessment of compliance with grid codes requirements can be challenging for researchers and (early stage) technology developers. To tackle this problem, a generic grid code is proposed in this article that defines certain requirements aimed for future scenarios with high shares of renewables. The proposed code is not linked to a particular grid of a certain region or country. The requirements are expressed in a mathematical way, in contrast to the legal format of real grid codes, to enable for easier implementation. The parameterised expressions enable for easy customisation for specific use cases. The compliance is assessed through reference under/over voltage test events and a test frequency disturbance, to which desired responses are specified. Detailed dynamic tolerance bands are defined around the desired responses, using linear parameterised functions providing a clear and continuous definition that leaves little room for interpretation. This generic grid code is inspired by the present regulations, especially the European grid code framework (ENTSO-E RfG) and the Irish grid code, which is seen by many as a benchmark regarding wind power integration. However, the generic grid code includes additional requirements that are not commonly part of grid codes today, including requirements covering asymmetric faults and inertia-like response to frequency deviations.
\end{abstract}

\section{Introduction}

Grid codes are technical specifications that define requirements mainly for generation units connected to electricity grids, including wind power plants (WPPs). Standardised grid codes are usually published by transmission system operators (TSOs) in most of the developed countries [1].

The writing style and organisation of grid codes as legal regulation documents usually differ significantly from other technical documents. Moreover, grid codes are lengthy documents with large numbers of pages such as the Irish grid code with $340+$ pages [2]. These issues pose challenges to researchers, either in

Email address: vrana@sintef.no (Til Kristian Vrana) 
academia or industry, and other stakeholders. However, some articles provide technical summaries/reviews [1,3], as an attempt to simplify grid codes formal documents to brief and technical-only articles.

The current research efforts in the field of grid codes are focused on compliance tests for different control methods $[4,5,6,7]$ and grid code reviews $[8,9]$ in order to summarize the requirements of several TSOs. Additionally, some researchers focus on market-oriented grid codes studies [10], particularly, the impact of ancillary services provision and retribution strategies to secure the necessary amount of support to ensure grid stability during disturbances, and the various methods to incentivise WPPs. However, and according to the authors' best knowledge, a generic grid code which is researcher-friendly, concise and purely technical version of a comprehensive grid code has not been proposed yet in the literature.

Grid codes can differ significantly across TSOs. This geographic diversity makes it challenging to get a clear and compact general overview on grid code requirements. The ENTSO-E aims to develop a uniform grid code framework for Europe [11, 12]. Although the new European grid codes provide a positive and encouraging step in the right direction, they still leave some key aspects unspecified, referring instead to regulation by the relevant TSO. Many research activities, including those focused on new wind turbine generator (WTG) control concepts, are in many cases not linked to a specific TSO. It is therefore unclear which grid code requirements could be used to assess potential grid code compliance, and it could be impractical at an early development stage to evaluate the compliance with every national and regional grid code. Hence, the proposed generic grid code could tackle this issue.

WPPs are increasingly facing similar requirements to conventional power stations, which is to some extent unavoidable, as the share of wind power in the generation mix is growing. The adaptation process of grid codes for WPPs key roles in system operation is not yet complete, and grid codes are expected to evolve further in the near future [1]. In the same context, some research work, including planning studies of WPPs integration is based on future scenarios. Hence, it is not clear which requirements should be used to represent future regulation.

To address these challenges, a generic grid code is proposed that defines a set of requirements, whereby it provides a tool to demonstrate compliance in future scenarios with high shares of renewables, without being tied to specific geographic locations. The proposed generic grid code mainly targets researchers and technology developers (e.g. wind turbine manufacturers). A preliminary vision of this generic grid code were presented in [13], but the set of requirements has evolved significantly since then. Although focused on wind power, the applicability of the grid code requirements for photovoltaics (PV) plants and battery energy storage systems (BESS) has also been briefly discussed in Section 3 of this article.

A framework for response requirements and tolerance bands for compliance assessment is defined as mathematical equations, continuous (i.e. linear) functions and block diagrams, to ensure consistency and facilitate its understanding. 
This approach also avoids the discontinuities provided by non-linear piece-wise expressions. Furthermore, using this mathematical way to express requirements offers advantages compared to the common practise, as it is short and precise, and as it leaves significantly less room for uncertain interpretations. This generic grid code framework is substantiated into specific requirements through a set of tabulated parameters. If necessary, the potential users of this generic grid code could customise the requirements to the power system under investigation by adjusting the relevant parameters. This mathematical approach could serve as an inspiration for the further developments of generic grid codes [14], where ENTSO-E could provide the equations and block diagrams with relating parameter ranges, while each TSO could set the parameters valid for his specific region within the provided range.

The main requirements provided are displayed in Figure 1. These requirements are inspired by the ENTSO-E grid code framework [11, 12] as well as the Irish grid code [2], which is seen by many as a good example regarding wind energy integration. A comparison with some of the key grid codes is given in table 1 .

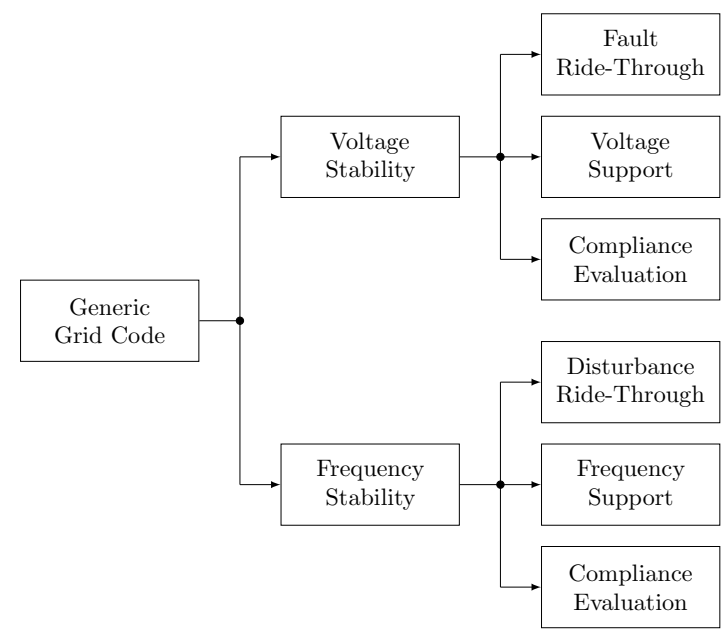

Figure 1: Overview of key requirements

The generic grid code defines advanced requirements such as the provision of synthetic/virtual inertia for frequency support and the inclusion of unbalanced fault response for voltage support during fault ride-through procedures. Both of these requirements are not commonly addressed in current grid codes, although their provision is reflected in the regulatory framework derived from ENTSO-E. The specifications also consider the incurred delays and filters which form a part of the measurement process to obtain the necessary signals to provide the aimed response.

In the light of the previous discussion and literature review, this article has three main contributions. First, the development of a generic grid code that facilitates compliance assessment decoupled from a specific geographic location 
Table 1: Grid codes comparison

\begin{tabular}{|c|c|c|c|}
\hline Grid Code & $\begin{array}{c}\text { ENTSO-E } \\
\text { RfG }\end{array}$ & EirGrid & $\begin{array}{c}\text { Generic } \\
\text { Grid Code }\end{array}$ \\
\hline Fault ride through requirement & specified & specified & specified \\
\hline Symmetric fault response & suggested & specified & specified \\
\hline Active/reactive current priority & not specified & active current & active current \\
\hline Asymmetric fault response & suggested & not specified & specified \\
\hline Sym./asym. current priority & not specified & not specified & equal \\
\hline Voltage/frequency support priority & not specified & implicit & voltage support \\
\hline Frequency disturbance ride through & specified & specified & specified \\
\hline Frequency disturbance response & suggested & specified & specified \\
\hline Frequency disturbance fast response & not specified & encouraged & specified \\
\hline Synthetic inertia & suggested & not specified & specified \\
\hline Transient compliance specification & not specified & $\begin{array}{c}\text { stepwise } \\
\text { partial }\end{array}$ & $\begin{array}{l}\text { continuous } \\
\text { complete }\end{array}$ \\
\hline
\end{tabular}

against the foreseen requirements that acknowledge the future challenges of high wind power shares in the generation mix. According to the authors' knowledge, this has not been comprehensively discussed in the literature yet. Second, the definition of advanced requirements such as unbalanced fault ride through, and synthetic inertia provision, which are commonly not acknowledged in detail in the current grid codes. The third contribution is the mathematical approach to response specification and compliance assessment with tolerance margins as continuous linear functions. This mathematical approach to express the requirements avoids discontinuities like piece-wise boundaries, and it is advantageous compared to the common practise, as it is short, precise and allows little room for interpretation.

In Section 2, voltage deviations are addressed, and in Section 3, frequency excursions are addressed. In Section 4, the conclusions of this work are given.

\section{Voltage-related Requirements}

Voltage support is a key requirement in grid codes to maintain voltage stability in the grid, mainly during disturbances, e.g. symmetric and asymmetric faults. In the event of a short circuit, the voltage will drop suddenly at the network buses which are in proximity to the fault location. A loss of load could also cause a sudden rise in voltage. Hence, generation units have to provide certain responses to enable quick and smooth recovery to nominal voltage. Moreover, the voltage level has to be maintained as close to nominal value as possible during and after the fault clearance. 


\subsection{Processing of voltage measurements}

Voltage measurement yields three phase voltages as instantaneous values. Based on these measured voltages, the positive and negative sequence voltages are derived. The positive sequence voltage $V_{1}$ is referred to as grid voltage. The negative sequence voltage $V_{2}$ is used additionally to determine the behaviour during asymmetrical faults, in both overvoltage and undervoltage situations.

Figure 2 shows the determination of the sequence voltages, which are the input parameters for Fault Ride-Through (FRT) requirement and control response. An application example of the RMS voltage calculation can be found in international standards as IEC [15] and the separation into positive and negative sequence $[16]$ (abc $\rightarrow 120$ ) could be performed in several ways, for instance as proposed in [17].

Table 2 is supplying the related parameters, which are based on [18].

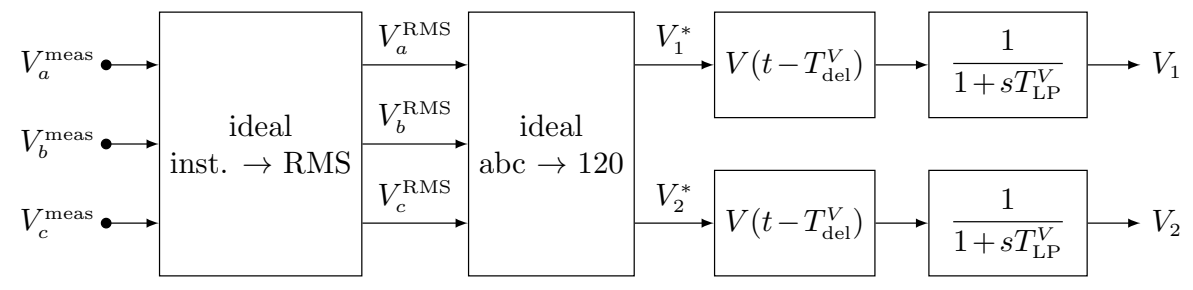

Figure 2: Processing of measured grid voltages

Table 2: Voltage measurement processing parameters

\begin{tabular}{l|ccc|l|l} 
Parameter & Min. & Recommended & Max. & Unit & Explanation \\
\hline$T_{\text {del }}^{V}$ & 0 & 0 & 20 & $\mathrm{~ms}$ & time delay \\
$T_{\mathrm{LP}}^{V}$ & 5 & 10 & 20 & $\mathrm{~ms}$ & filter time constant
\end{tabular}

For compliance assessment, the WTG current in the sequence domain should be determined in the same way as described here for the voltage.

\subsection{Operational Range of the Grid Voltage}

The operational range of the grid voltage, bounded by $\left[V_{1, \mathrm{lim}-}^{\mathrm{norm}}, V_{1, \mathrm{lim}+}^{\mathrm{norm}}\right]$, is defined as a $\pm 15 \%$ tolerance around the nominal grid voltage $V_{\text {nom }}$. The relating parameters are specified in Table 3. This is taken from the ENTSO-E grid code [11] for the continental European synchronous area for voltage levels below $300 \mathrm{kV}$. The specified time limitation is omitted in the proposed generic grid code, stating that operation within this range has to be possible on a continuous basis. This is based on the thought that the specified time intervals are longer in comparison to all relevant time constants, indicating that they only affect steady state operation. The continental European synchronous area was chosen, as it has the strictest requirements. In the proposed generic grid code, this characteristic is valid for all voltage levels and all synchronous areas. 


\subsection{Fault-Ride-Through Requirements}

The ENTSO-E RfG [11] specifies a range of possible FRT requirements, within which the relevant TSO may demand undervoltage-fault withstanding capability. In the proposed code, the strictest requirement within the range is considered. Overvoltage-faults are not treated in ENTSO-E, so the related requirement of the generic grid code is designed based on the expected response of conventional generators. The related parameters are specified in Table 3, while the FRT curves are displayed in Figure 3.

Table 3: FRT curve parameters

\begin{tabular}{l|c|l|l} 
Parameter & Value & Unit & Explanation \\
\hline$V_{1, \text { lim }+}^{\text {fault }}$ & 1.35 & $\mathrm{pu}$ & upper extended operation limit \\
$V_{1, \text { lim }+}^{\text {norm }}$ & 1.15 & $\mathrm{pu}$ & upper normal operation limit \\
$V_{\text {nom }}$ & 1.0 & $\mathrm{pu}$ & nominal voltage \\
$V_{1, \text { lim- }}^{\text {norm }}$ & 0.85 & $\mathrm{pu}$ & lower normal operation limit \\
$V_{1, \text { lim- }}^{\text {fault }}$ & 0 & $\mathrm{pu}$ & lower extended operation limit \\
\hline$V_{2, \mathrm{lim}}^{\text {fault }}$ & 0.5 & $\mathrm{pu}$ & neg. seq. extended operation limit \\
$V_{2, \mathrm{lim}}^{\text {norm }}$ & 0.05 & $\mathrm{pu}$ & neg. seq. normal operation limit
\end{tabular}



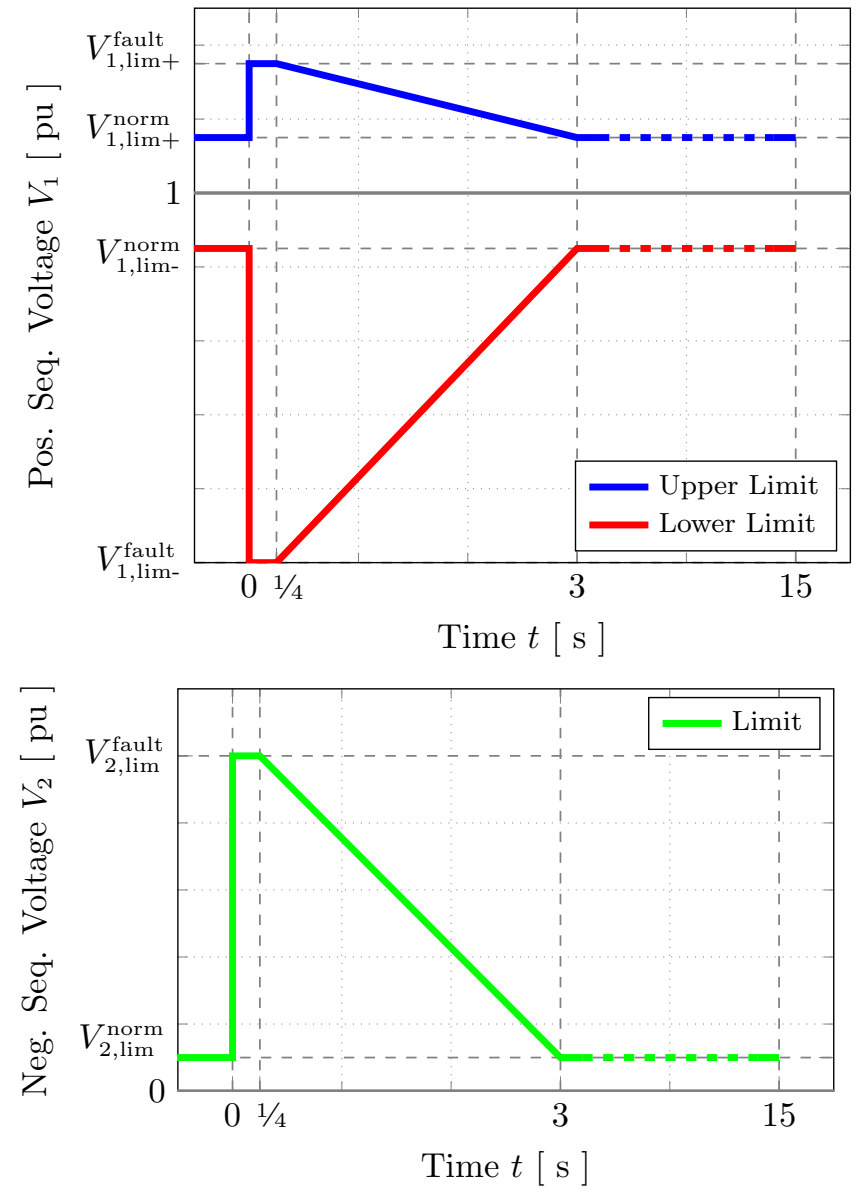

Figure 3: Fault ride through requirement

The FRT requirements refer to the maximum and minimum $V_{1}$ and $V_{2}$ sequences. This is an aspect of novelty and ensures the comprehensive nature of the proposed generic grid code, as a simplified approach that considers positive sequence $V_{1}$ only could lead to problems. For instance, single phase overvoltage are not necessarily detectable in the positive sequence, especially when the pre-fault voltage, maintained on the other two healthy phases, was rather at the lower end of the operational range. However, grid-connected assets should react to counteract such an overvoltage (with a reaction in the negative sequence), or at least they should be allowed to disconnect in such a case.

Two sequential events are considered as separate, if there is at least $15 \mathrm{~s}$ between them, meaning that the voltage needs to be at least $12 \mathrm{~s}$ between $V_{\mathrm{lim}-}^{\mathrm{norm}}$ and $V_{\lim +}^{\text {norm }}$ before a new event must be tolerated. This can be seen in Figure 3 . 


\subsection{Response towards Voltage Changes}

The required control response towards voltage disturbances in the grid is described in this subsection. Reactive current is selected (rather than power) as the compliance indicator of the applied control response. Reactive power could be also a reasonable alternative, as it is used in some grid codes and generally referred to in the electric power industry. However, the measurements and calculation of power during the very early stages of fault and post-fault could be challenging, making the utilisation of the directly measurable current more practical and meaningful. The conceptual representation of the determination process for current references as a function of the voltage measurements and calculations are depicted in Figure 4 and the corresponding parameters are summarised in Table 4.

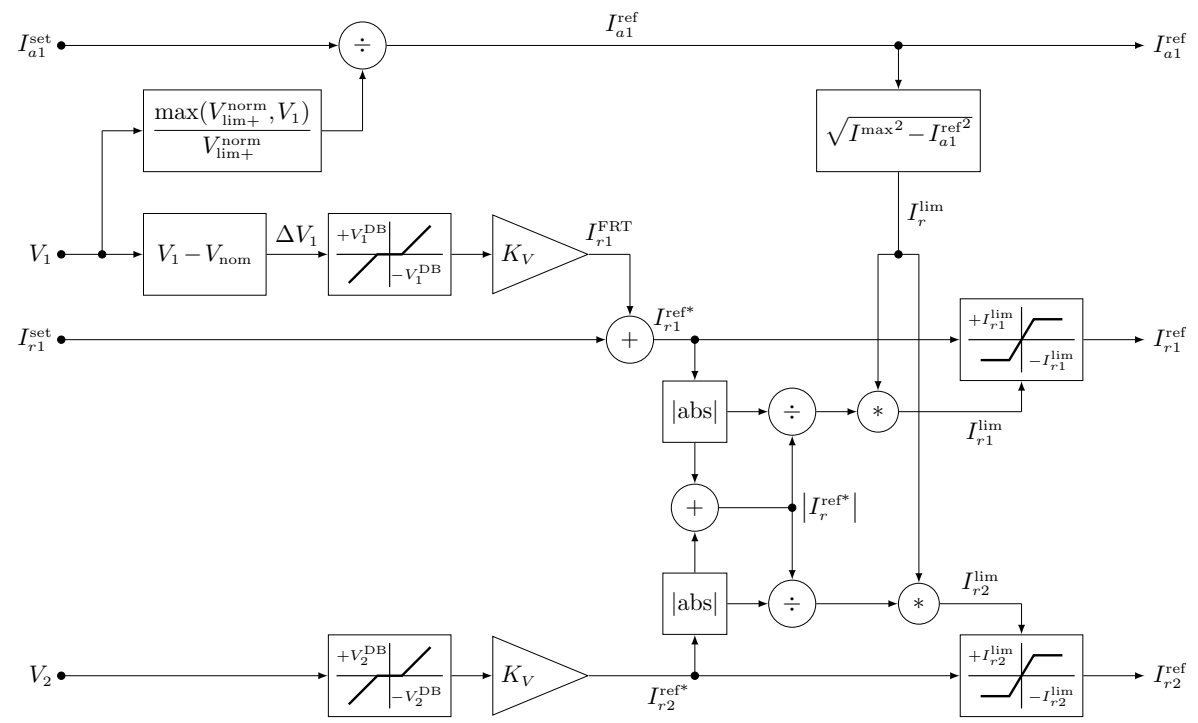

Figure 4: Block diagram of the voltage support

Table 4: Voltage support parameters

\begin{tabular}{l|c|l|l} 
Parameter & Value & Unit & Explanation \\
\hline$V_{1}^{\text {DB }}$ & \pm 0.15 & $\mathrm{pu}$ & pos. seq. dead band \\
$V_{2}^{\text {DB }}$ & \pm 0.05 & $\mathrm{pu}$ & neg. seq. dead band \\
$K_{V}$ & -3.0 & - & droop constant \\
$I^{\max }$ & 1.32 & $\mathrm{pu}$ & maximum current
\end{tabular}

The active component of the positive sequence current $I_{a 1}$ changes not more than necessary. This is done in order to limit the impact on the active 
power balance of WTGs and the grid. During undervoltage conditions, it remains unchanged $\left(I_{a 1}^{\mathrm{ref}}=I_{a 1}^{\text {set }}\right)$, which is inspired by $[2,19]$. During overvoltage conditions, it is scaled down according to the voltage rise beyond the upper limit for continuous operation, to maintain constant active power. This is implemented through the 'max' control block in the upper left corner of Figure 4.

The reference for the active component of the negative sequence current is always zero $\left(I_{a 2}^{\mathrm{ref}}=0\right)$, and does not depend on the grid voltage. The reactive current references are functions of the voltages, for both positive and negative sequence. The WTG is expected to be capable of delivering reactive current of at least $50 \%$ of the active current. The converter needs to be rated according to the maximum achievable current $I^{\max }$, which is significantly higher than the nominal current. Such high current rating is necessary to maintain full power even at reduced voltage, and in order to allow the system to deliver reactive current even when the WTG is operating at full active current. Considering the minimum allowed grid voltage $\left(V_{1, \mathrm{lim}-}^{\mathrm{norm}}=0.85 \mathrm{pu}\right)$, active current at full power could reach $1 / 0.85=1.18$ pu under normal operating conditions. The requirement of an reactive current capability of $50 \%$ of the active current, leads to a minimum value for $I^{\max }$ :

$$
I^{\max }=\sqrt{\left(\frac{1}{0.85}\right)^{2}+\left(\frac{0,5}{0.85}\right)^{2}}=1.32 \mathrm{pu}
$$

Saturation blocks define the difference between the ideal instantaneous response $\left(I_{r 1,2}^{\mathrm{ref*}}\right)$ and the actual reference to be applied $\left(I_{r 1,2}^{\mathrm{ref}}\right)$. The active current still has priority, in order to limit the impact on the active power of the wind generation, in order to avoid triggering generation-demand imbalance issues in the grid. The prioritisation usually relies on the local voltage support and global (system wide) frequency support. Voltage dips induced frequency excursions, are to some extend unavoidable, even with priority on active current, as maintaining the active power setpoint at lower voltage is not feasible due to current restrictions. A loss of large offshore WPP due to nearby fault will make the grid suffer a significant loss of generation. For example, the threshold reserve in central Europe grid is still considered as $2 \times 1.5 \mathrm{GW}$, which is the installed capacity of two large nuclear power plants. Hence, the sudden loss of this amount of wind power could trigger a frequency event, if the grid code does not require sustaining the active current within the achievable limits during the event. It is worth mentioning that, the Irish grid code implemented active current priority a long time ago, due to the small size of the Irish grid and the extensively growing share of wind power in the generation mix. Hence, the active current priority is proposed as a countermeasure in this future-oriented generic grid code. This means that the upper limit of the reactive current saturation depends on the magnitude of the active current $I_{a 1}^{\mathrm{ref}}$. There is no prioritisation of positive or negative sequence, hence, when the desired reactive current exceeds the technical possibilities, both sequences are reduced to stay within the limits, while maintaining the ratio between the sequences. The references in positive and negative sequence are then added up after park transformation in order to 
obtain the corresponding setpoints. The calculation procedure could be selected by each manufacturer, depending on their control system strategy, and is not imposed by the grid code; an application example can be found in [20]. It is of note that in case of DFIG machines, the distribution between the amount of current provided by the stator and the converter is regulated according to the capacity of the installed converter (i.e. normally $30-40 \%$ of the full rating of the WTG). The exact implementation is decided by the manufacturer, while the grid code considers the total current injected only.

\subsection{Tolerance Band}

There is a possibility that a real WPP might not respond perfectly with respect to the grid code requirements, due to non-linearities, model simplifications, controller limitations, measurement noise, etc., the compliance assessment with the grid code requirements should allow certain deviations within reasonable limits. A tolerance band that envelopes the ideal response is proposed. In order to avoid discontinuities, a block diagram to compute the tolerance band as a continuous function of the voltage is defined in Figure 5. The tolerance bands are different for positive and negative sequence currents, and they are calculated using the corresponding sequence voltage. The parameters that regulate the tolerance bands evolution are expressed in Table 5 .

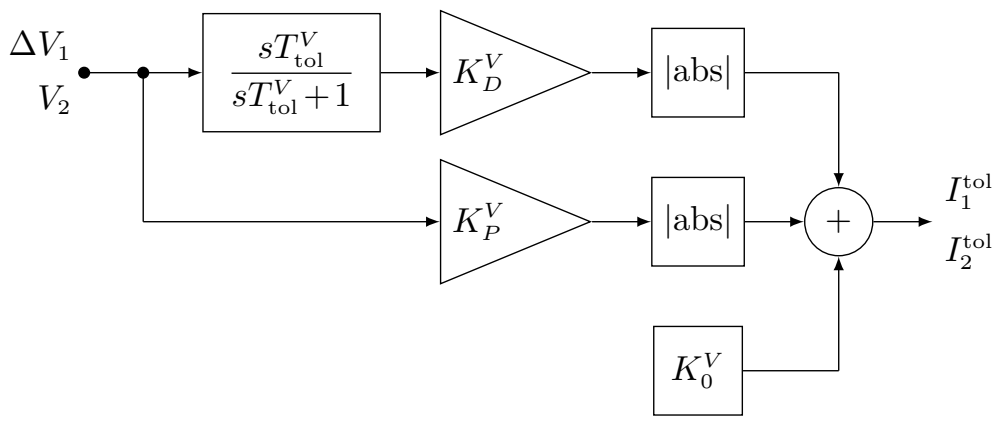

Figure 5: Calculation of the voltage support tolerance band

Table 5: Parameters of the voltage support tolerance band

\begin{tabular}{l|c|l|l} 
Parameter & Value & Unit & Explanation \\
\hline$T_{\text {tol }}^{V}$ & 40 & $\mathrm{~ms}$ & filter time constant \\
$K_{D}^{V}$ & 2.5 & - & derivative gain \\
$K_{P}^{V}$ & 0.2 & - & proportional gain \\
$K_{0}^{V}$ & 0.1 & $\mathrm{pu}$ & base tolerance band constant
\end{tabular}

The tolerance band is calculated based on continuous functions, however, the voltage support is only triggered when the voltage deviation violates a 
predetermined deadband, as shown in Figure 4. The tolerance band gets wider according to the severity and the time-gradient of the voltage deviation. This accounts to the fact that regulating the currents is more challenging at these stages of the fault. Actually, phase-angle jumps usually occur during these stages of the fault, which limit the ability of the generation unit to provide the required FRT response, to comply with the grid code requirements due to the momentary loss of synchronization. Hence, the proposed dynamic widening of the tolerance bands provides a time window where the unit detects the new frequency and phase-angle to trigger the required response by the grid code. The response of a WTG/WPP is considered compliant with the grid code requirement, if it remains within the tolerance band, as illustrated in Equation (2).

$$
\begin{aligned}
\left|I_{a 1}^{\mathrm{ref}}(t)-I_{a 1}(t)\right| & \leq I_{1}^{\mathrm{tol}}(t) \\
\left|I_{r 1}^{\mathrm{ref}}(t)-I_{r 1}(t)\right| & \leq I_{1}^{\mathrm{tol}}(t) \\
\left|I_{r 2}^{\mathrm{ref}}(t)-I_{r 2}(t)\right| & \leq I_{2}^{\mathrm{tol}}(t) \\
\left|I_{a 2}(t)\right| & \leq I_{2}^{\mathrm{tol}}(t)
\end{aligned}
$$

\subsection{The test fault}

The considered test fault (depicted in Figure 6) shows the evolution of the grid voltage in phase domain in RMS. The figure also shows the positive sequence FRT curve (in dashed line) where the WTG should remain connected and provide voltage support. This test fault can be used for compliance assessment, and the compliant response of a WTG is shown in Subsection 2.7. As seen, the selected fault is not the most severe fault that the system must withstand since in the initial short-term fault voltage range $[0-1.35 \mathrm{pu}]$ the plant must remain operational.

This test fault is designed to check the behaviour of the system during strong transient and during smooth transitions in the same test. It also represents the profile requested by many European grid codes nowadays for FRT operations where there are contemplated the initial fault transient, the second transient of the fault clearance and the progressive recovery to normal operation.

Figure 6 represents the test fault curves in phase domain which is analysed in both the positive and negative sequence and depicted in Figure 7 . That represents the same voltage profile as in phase domain but adding to the analysis the unbalance of the grid voltage. This is achieved by applying a sequence filter as shown in Figure 2.

The curves displayed in Figure 7a represent three different types of test faults: single-phase (cyan line), two-phase (magenta line) or symmetrical three-phase (green line) fault; both cases are included under- and overvoltage. As seen, the voltage magnitudes show a great variability depending on the number of phases affected by the fault, therefore the voltage support response should be done accordingly.

The negative sequence is depicted in Figure $7 \mathrm{~b}$ for the same fault situations as previously described. The negative sequence is an indicator of the voltage 


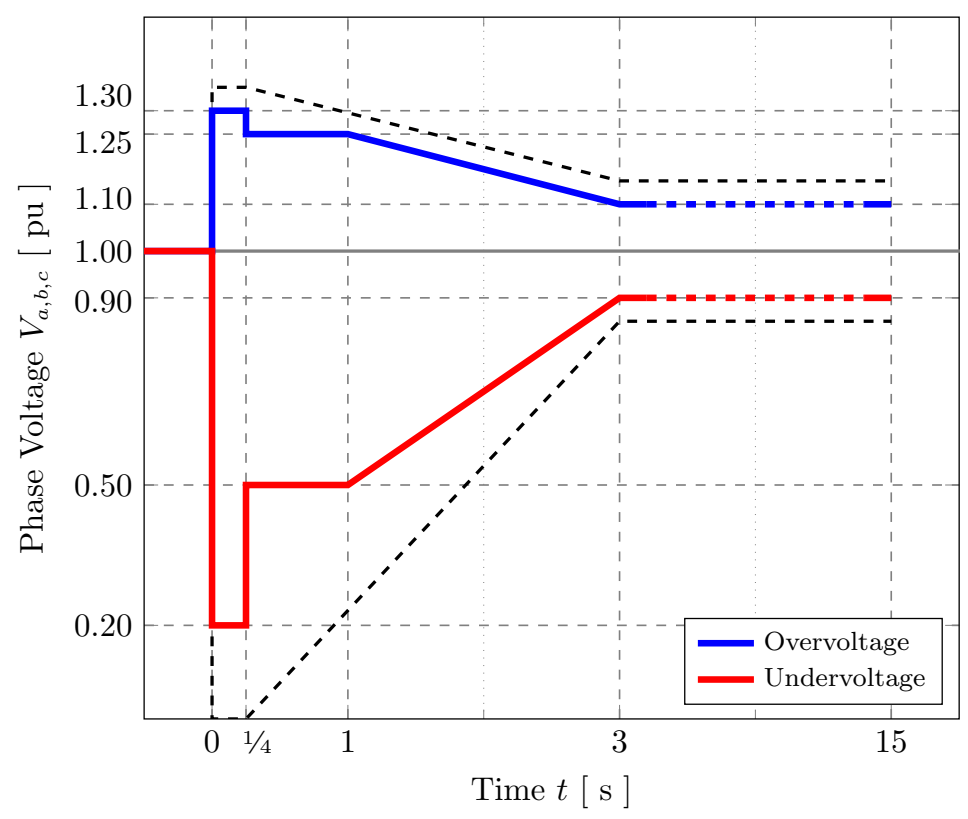

Figure 6: Test fault (dashed lines show the FRT operational range)

unbalance that the network suffers during the fault. In particular, the absolute minimum is zero (perfectly balanced grid) where the three phases have the exact same voltage. In consequence, the negative sequence voltage increases as the voltage at the three phases dynamically change to be more unbalanced, but it does not have a negative value. Hence, only three curves are displayed in Figure $7 \mathrm{~b}$, the negative sequence value is exactly the same either is an overvoltage or an undervoltage situation and, also, for single- or two-phase faults if the faulty phases retain the same voltage. Meanwhile, during symmetric faults the negative sequence is zero since there is no voltage difference among phases. 


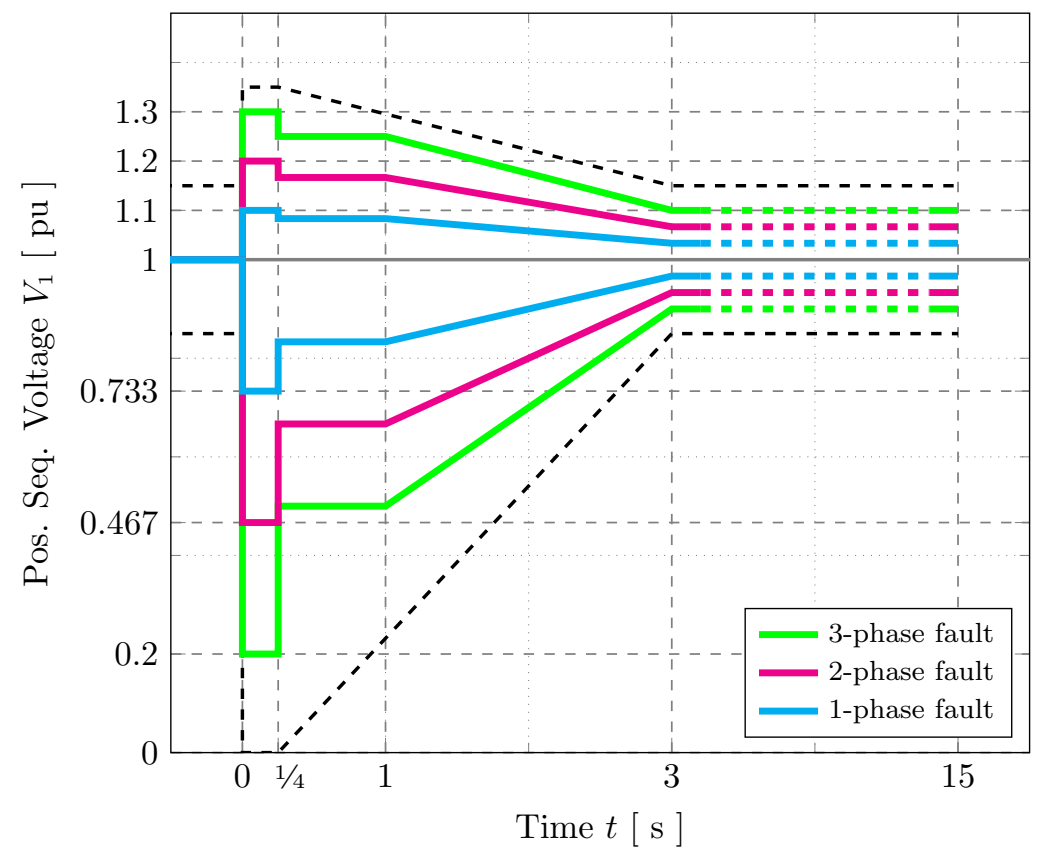

(a) Positive sequence

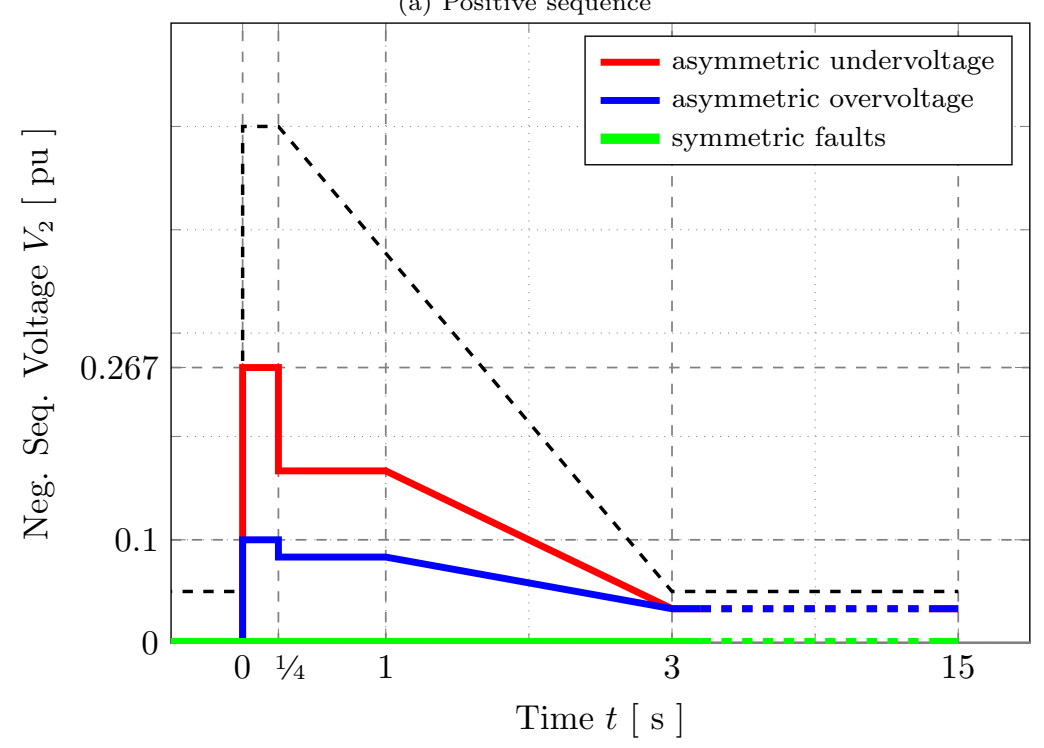

(b) Negative sequence

Figure 7: Test Faults in Sequence Domain

\subsection{Response towards the Test Fault}

An example of the compliance analysis is shown in Figure 8 for a single-phase undervoltage fault and in Figure 9 for a three-phase overvoltage situation. In 
these plots, the evolution of the tolerance bands, the ideal responses and a simulated response of a WTG model are shown. The depicted data corresponds to the test fault shown in Figure 6, applied to the controllers shown previously where it is assumed that the WTG is already generating its rated output $\left(I_{a 1}=1\right)$, which is a challenging scenario.

\subsubsection{Ideal response}

The active current is required to be constant in an undervoltage fault (Figure 8a) in order to avoid exceeding the converter operational limits. During the overvoltage incident, the active current is allowed to be reduced (Figure 9a) in order to maintain the output power almost constant, reducing the stress on the mechanical structure of the turbine. The reactive current injection shall counteract the voltage deviation, making the sign of the reactive current injection a function of the sing of the voltage deviation. It can be observed how the reactive power is produced when a voltage drop is detected (Figure 8b), while it is absorbed by the WTG when the voltage rises above its nominal value (Figure 9b).

Moreover, during an asymmetric event, negative sequence reactive current injection is required (Figure 8c). As observed in Figure 8, the negative sequence compensation that provides support in unbalanced situations acts in parallel to the reactive current positive sequence injection since both actions should happen simultaneously in order to comply with the required voltage support. However, when the event is symmetric (three-phase fault), this negative sequence response is not triggered (Figure 9c).

\subsubsection{Tolerance band}

The differential part of the tolerance band calculation shows steep surges following events of fast changing voltages, which commonly occur at the beginning of the fault and after the fault clearance, as shown in Figure 8 and Figure 9. During these very steep and fast overshoots, the tolerance band is widened, in practise realising a time delay to the requirements; almost no specific behaviour is enforced in the very first moment after both fault occurrence and clearance. Hence, the proposed generic grid code considers the difficulty of precise current control during such fast transients in real systems.

\subsubsection{Compliance of a typical wind turbine generator}

The grid code compliance studies are usually carried out to examine the asset response to the test events provided in the grid code. In this article, the voltage at the connection point of the WTG follows exactly the event voltage profile. This is achieved by using a programmable grid model in MATLAB/Simulink. Similarly, the frequency pattern of the test event is directly applied to the frequency signal input to the supplementary controllers which enable the WTG controls, where the WTG frequency support is assessed against the required active power response as explained later in Section 3. Actually, many TSOs apply the same approach to provide compliance certificates for newly connected generation units. 
The proposed control method is applied to the benchmark Type 3 DFIG model available in the MATLAB Simscape to examine its compliance with the proposed generic grid code. It should be mentioned that, the grid short-circuit capacity at the fault point should not affect the compliance test, as the grid model is already programmed to imitate the voltage profile of the grid code event (shown in Figure 6) regardless the generator response. This is the most practical way to evaluate the compliance fairly without the interference of any other factors. The analysis of the impact of generator response on the power system behaviour during certain events at various operation conditions is a different type of studies (i.e. power system dynamic stability). This type of studies consider the impact of special controls that enable renewable generators to provide ancillary services to the grid, and its impact on the grid voltage and frequency responses compared to the original response (i.e. when the renewable generator does not contribute to ancillary services).

The $d q 0$ frame of the WTG converter is driven by the proposed controller. The signals of the proposed grid code compliant simple control are processed to match the conventional DFIG dq-frame control for the simulation cases. It is worth mentioning that this article is focused on the development of the proposed generic grid code, not the optimised integration of the proposed supplementary controller into the conventional controls of WTGs. These simulations should demonstrate that typical WTGs can comply, meanwhile better compliance could be achieved by Type 4 WTGs due to the presence of the full rated power electronics converter (PEC) which provides more flexible control. The incident wind speed is set to $15 \mathrm{~m} / \mathrm{s}$ to ensure that the WTG is providing its rated output before the occurrence of the examined voltage events. This is the most common scenario in the literature where FRT methods are always tested at a $1 \mathrm{pu}$ output to ensure their robustness, and exploit how the WTG could handle this challenging situation during the fault.

The supplementary control is activated once $V_{1}$ violates the safe deadband and stays operational until the voltage recovers and settles within the deadband for a few seconds. The rated power of the integrated PEC of the WTG is $40 \%$ of its rated power.

This section shows the response to two events: 3-phase overvoltage and unbalanced (1-phase) undervoltage. To maintain a reasonable article length, the authors do not include the balanced (3-phase) fault. The 3-phase fault showed very high alignment with the ideal response, and the authors thought it could be more interesting to include the WTG reactions to the other two events.

As seen in Figure 8 and Figure 9, the WTG shows reasonable compliance in the two examined events. However, after the voltage recovers to the safety deadband, some deviation from the ideal response is observed. For example, there is a slight deviation from ideal response of the active current positive component (Fig 9-a) as the voltage recovers to the safe deadband. This could be due to the deactivation of the voltage support controller when the voltage settles within the deadband, hence, the WTG is driven by its conventional control. In addition, the induction machine dynamics and the rating of the PEC play a role in the process. 
It is also noted that the reactive current injected by the WTG into the grid, achieves very high compliance with the ideal response during the most critical intervals of the two events. These intervals are of top priority for TSOs. Moreover, the WTG has successfully maintained a reasonable active current during both events, which contributes to the smooth recovery of power generation after fault clearance, mitigating the possibility of initiating a frequency event due to power imbalance. 


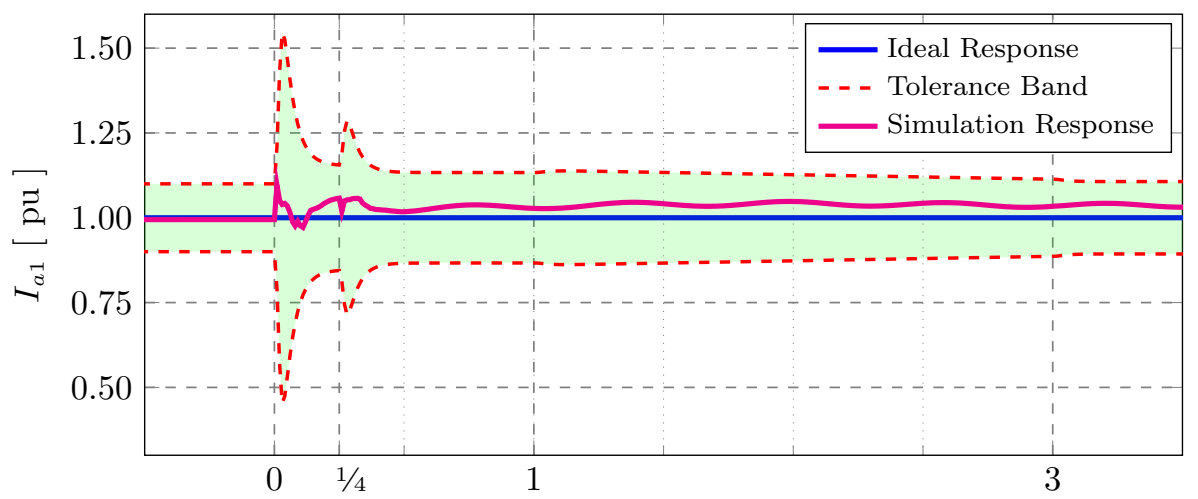

(a) Positive sequence active current

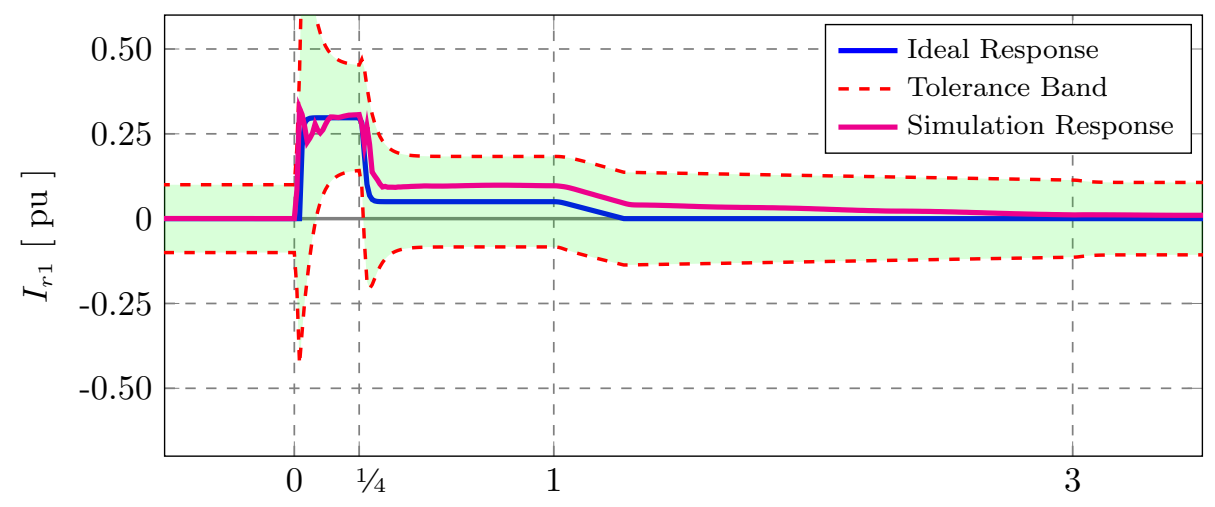

(b) Positive sequence reactive current

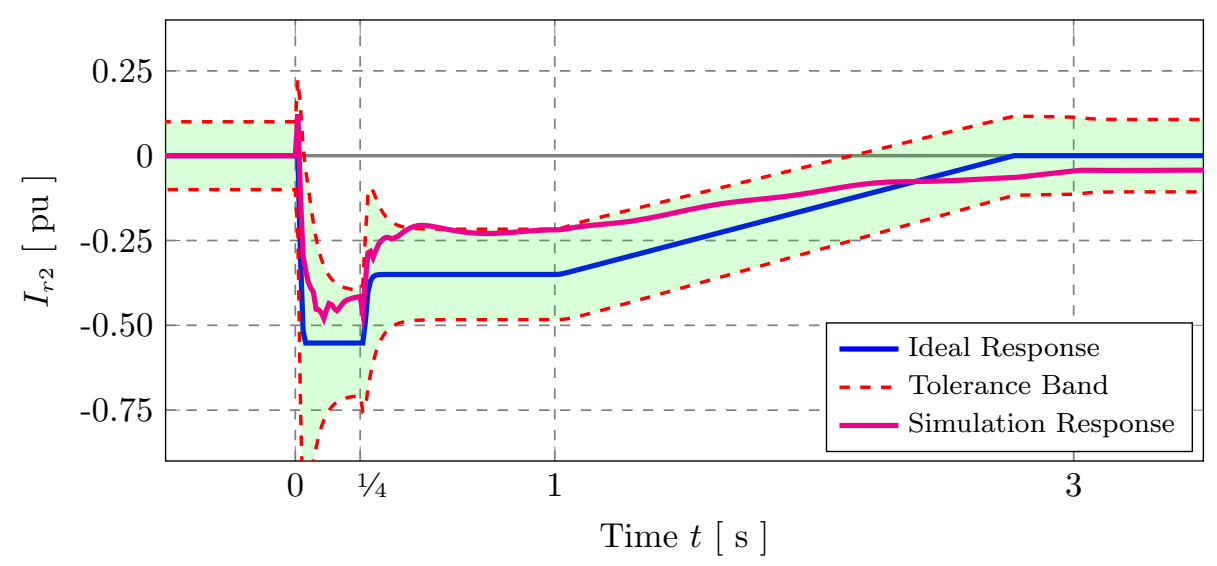

(c) Negative sequence reactive current

Figure 8: The response towards a single-phase undervoltage 


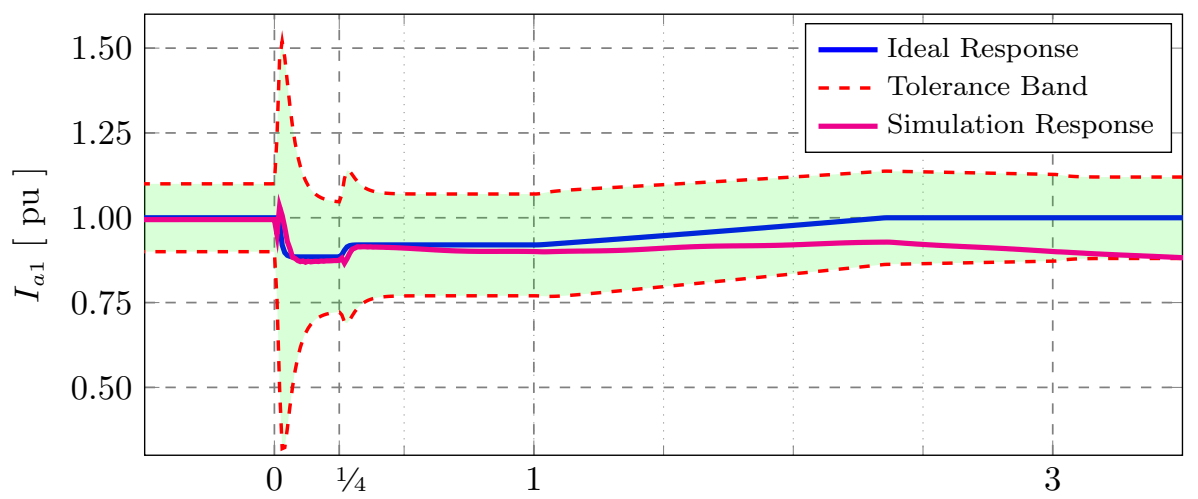

(a) Positive sequence active current

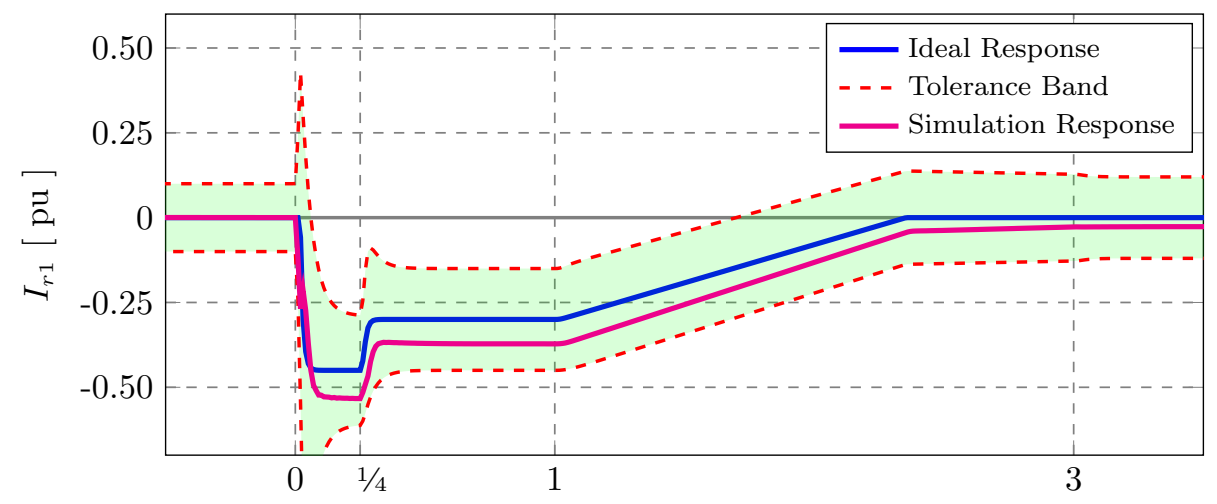

(b) Positive sequence reactive current

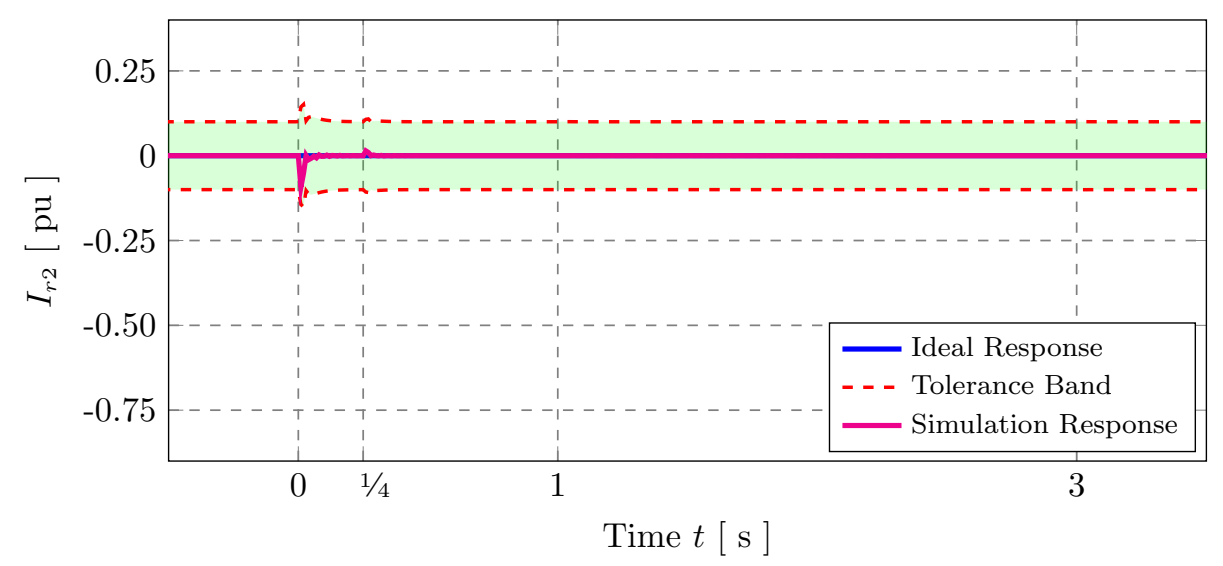

(c) Negative sequence reactive current

Figure 9: The response towards a three-phase overvoltage 


\section{Frequency-related Requirements}

Modern WTGs are decoupled from the grid frequency dynamics via the PEC, hence, the process of frequency support provision incorporates two stages, frequency measurement and active power control to provide the aimed support. This section describes the proposed frequency support requirements according to the current practices relying on the common measurements approaches and proposed support methods. As voltage dynamics are much faster than frequency dynamics, and as voltage deviations are generally of shorter duration than frequency excursions, it is acceptable and expected to prioritise voltage support described in Section 2 over the frequency support. In practise, this means that the frequency support is mandatory only if the voltage is within the regular operation range. Particularly, when a short circuit event happens during a frequency event, frequency support should be adapted or halted for a short interval, to enable full voltage support during the fault. This article is focused on the grid codes of large power systems. However, it should be mentioned that island power systems of relatively smaller inertia and limited spinning reserve could be more vulnerable to frequency events under operation with high shares of wind energy. In addition, some aspects of the proposed generic grid code are inspired by the Irish grid code, which represents a bright example regarding integration of wind power into power grids of relatively small installed capacity compared to larger grids like UK National Grid or the Spanish grid.

\subsection{Processing of the Grid Frequency Measurement}

Measurement challenges include communication delays and signal noise, where frequency is measured at the Point of Common Coupling, which is also called Connection Point in some literature. The Phase-Locked Loop (PLLs) are commonly used to synchronise the grid-side converters with the grid, where the frequency measurement signal is also used as an input to the supplementary controllers that are integrated to provide frequency support [21]. However, TSOs are expanding the integration of Phasor Measurement Units (PMUs) at the key buses of the transmission systems, which should contribute to the integration of more smart control methods leading to larger grid flexibility. The frequency measurement is communicated it to the supplementary frequency support controllers of the WTGs or the holistic controller of the WPP [22, 23, 24]. The delay caused by such process is between $20-30 \mathrm{~ms}$ relying on the quality of the measurement equipment and possible interference of other factors [25]. The model depicted in Figure 10 represents the common measurement process taking into account potential delays. The relevant parameters are given in Table 6 .

In addition, a low-pass filter is integrated to avoid undesirable noise and responses to very fast frequency oscillations. The signal of frequency deviation $(\Delta \mathrm{f})$ could be an input to compute the frequency support controller response of power-electronics-dominated generation (e.g. renewable energy and energy storage systems) $[26,27]$. 


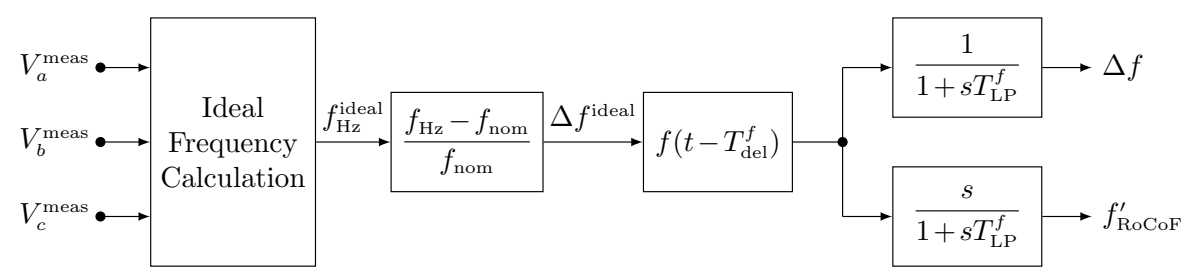

Figure 10: Measurement of the grid frequency

Table 6: Frequency measurement parameters

\begin{tabular}{l|ccc|l|l} 
Parameter & Min. & Recommended & Max. & Unit & Explanation \\
\hline$f_{\text {nom }}$ & 50 & 50 & 50 & $\mathrm{~Hz}$ & nominal frequency \\
$T_{\mathrm{del}}^{f}$ & 0 & 0 & 40 & $\mathrm{~ms}$ & time delay \\
$T_{\mathrm{LP}}^{f}$ & 10 & 20 & 40 & $\mathrm{~ms}$ & filter time constant
\end{tabular}

\subsection{Operational Range of the Grid Frequency}

A WPP should be able to operate at frequencies as low as $49 \mathrm{~Hz}$ on a continuous basis, and down to $47.5 \mathrm{~Hz}$ for a limited time where below $49 \mathrm{~Hz}$ the load shedding protocols are activated, and below $47.5 \mathrm{~Hz}$ the generators under-frequency relays are allowed to trip according to ENTSO-E Policy 5: Emergency Operations. It has to tolerate frequency gradients of up to $2 \mathrm{~Hz} / \mathrm{s}$ [12]. This defines the disturbance ride through characteristic, as displayed in Figure 11. The generic frequency disturbance event, displayed in Figure 11, is used to assess the compliance of a certain WTG model where two common support methods are examined.

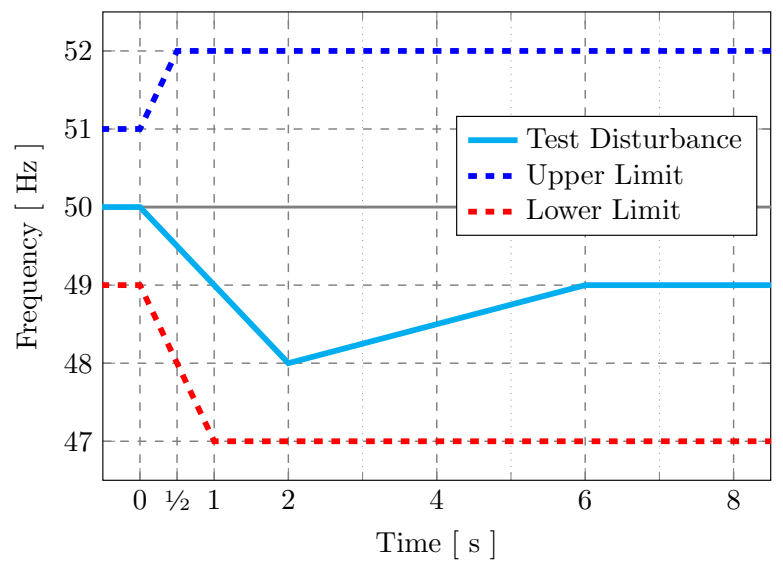

Figure 11: Active power requirements and test event 
Table 7: Active power requirements and test event parameters

\begin{tabular}{l|l|l|l} 
Parameter & Value & Unit & Explanation \\
\hline$f_{\text {lim }+}^{\text {dist }}$ & 52 & $\mathrm{~Hz}$ & upper extended operation limit \\
$f_{\text {lim }+}^{\text {norm }}$ & 51 & $\mathrm{~Hz}$ & upper normal operation limit \\
$f_{\text {nom }}$ & 50 & $\mathrm{~Hz}$ & nominal frequency \\
$f_{\text {lim- }}^{\text {norm }}$ & 49 & $\mathrm{~Hz}$ & lower normal operation limit \\
$f_{\text {lim- }}^{\text {dist }}$ & 47 & $\mathrm{~Hz}$ & lower extended operation limit
\end{tabular}

\subsection{Response towards Frequency Changes}

The developed frequency code requirements are based on the response of the proposed PD controller, which means that a compliant response should be in line with the response of the proposed supplementary controller. The controller is shown in Figure 12 and the parameters are in Table 8. This PD controller provides power surge $\left(P_{\text {ref }}^{\mathrm{PD}}\right)$, which is composed of two components: one relies on frequency deviation, and the other is proportional to the RoCoF. The proportional part reflects the response of conventional synchronous generator with a certain droop (R), which helps TSOs to maintain the traditional system dynamics during frequency excursions, at high shares of wind power. The differential component of the controller mimics the natural inertia response of a typical synchronous generator rotating parts.

The proportional frequency support is triggered when the frequency deviation exits the deadband, but the differential part is always active (as shown in Figure 12). The deadband could be also applied to the differential component in a power system with sufficient natural inertia, but this future-oriented grid code considers the scenario of low system inertia. In the extreme scenario of a $100 \%$ power electronics-dominated power systems, where all power converters have a deadband around the nominal frequency, no stable operating point may exist within the deadband. The impact of deadband width has been considered in [28].

The TSOs do not apply certain control techniques to achieve the compliance, hence the WPPs operators could apply their preferred control methods according to their techno-economic studies. This could be similar to the proposed controller or something different where the implementation of rate of change of frequency (RoCoF) signal as one of the controller inputs is not mandatory. In particular, any control design is compliant as long as it provides a response that is within the required tolerance band. However, using RoCoF will enable an early detection of frequency disturbances.

The proposed response and limits are given in per unit, and the outcome is multiplied by the pre-event generation magnitude $\left(P_{\text {set }}\right)$, making the control response relative to the actual power production preceding the disturbance. This is a different approach compared to conventional grid codes where the power step/surge is assessed against the rated power of the generator. In particular, 


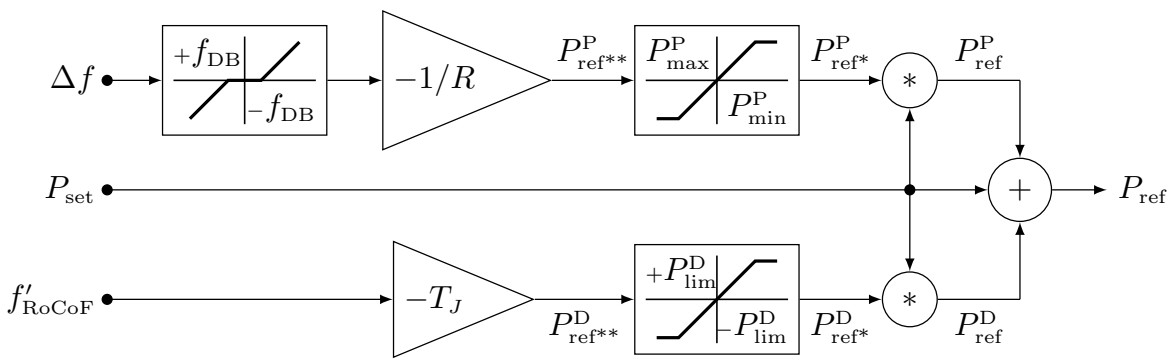

Figure 12: Block diagram of the frequency support requirement

Table 8: Frequency support parameters

\begin{tabular}{l|c|l|l} 
Parameter & Value & Unit & Explanation \\
\hline$f_{\mathrm{DB}}$ & \pm 20 & $\mathrm{mHz}$ & frequency dead band \\
& \pm 0.0004 & $\mathrm{pu}$ & \\
$R$ & 4 & $\%$ & primary droop constant \\
$T_{J}$ & 10 & $\mathrm{~s}$ & inertia time constant \\
$P_{\mathrm{lim}}^{\mathrm{D}}$ & \pm 0.1 & $\mathrm{pu}$ & derivative component \pm limits \\
$P_{\max }^{\mathrm{P}}$ & +0.1 & $\mathrm{pu}$ & porportional component upper limit \\
$P_{\min }^{\mathrm{P}}$ & -0.8 & $\mathrm{pu}$ & proportional component lower limit
\end{tabular}

the conventional approach is impractical for wind power, taking into account the varying nature of wind speed, where WPPs provide their nameplate capacity for only about $10-15 \%$ of the time $[29,30]$. Moreover, the RoCoF-based response component requires a power increase within a very short duration, which could be challenging for WTGs due to the rate of change limits on active power set-points implemented by WTG conventional controls. To provide this short-term power injection, it could be useful to slow down the WTG to extract the available stored kinetic energy in the rotating parts of the WTG, which is a widely investigated concept in the literature. The latter concept is usually called virtual or synthetic inertia, where the WTG emulates the natural inertia response of synchronous generator through certain supplementary control loops which adjust the active power setpoint based on the input frequency measurement. However, even using the deloading support method only, the WTG could be able to provide both the synthetic inertia as well as primary reserve due to the responsive nature of the PEC that couple the WTG to the grid. Most of the proposed controls in the literature apply a common approach, where the virtual inertia component is dependent on the RoCoF as an input signal meanwhile the primary support component relies on frequency deviation [31]. 


\subsection{Tolerance Band}

Since a real WPP may not offer a perfect response due to wind speed variations, non-linearities, model simplifications, measurement noise, controller limitations, etc., the compliance with grid code requirements allows certain deviations within reasonable limits. A tolerance band that envelopes the desired reference value is implemented. In addition, it is challenging for renewable energy sources, except hydropower and without the support of energy storage, to provide constant response over time. The tolerance band also considers this mitigation. In order to avoid discontinuities, a block diagram to compute the tolerance band as a continuous function of the frequency and RoCoF is defined in Figure 13. The parameters regulating the tolerance bands evolution are mentioned in Table 9 .

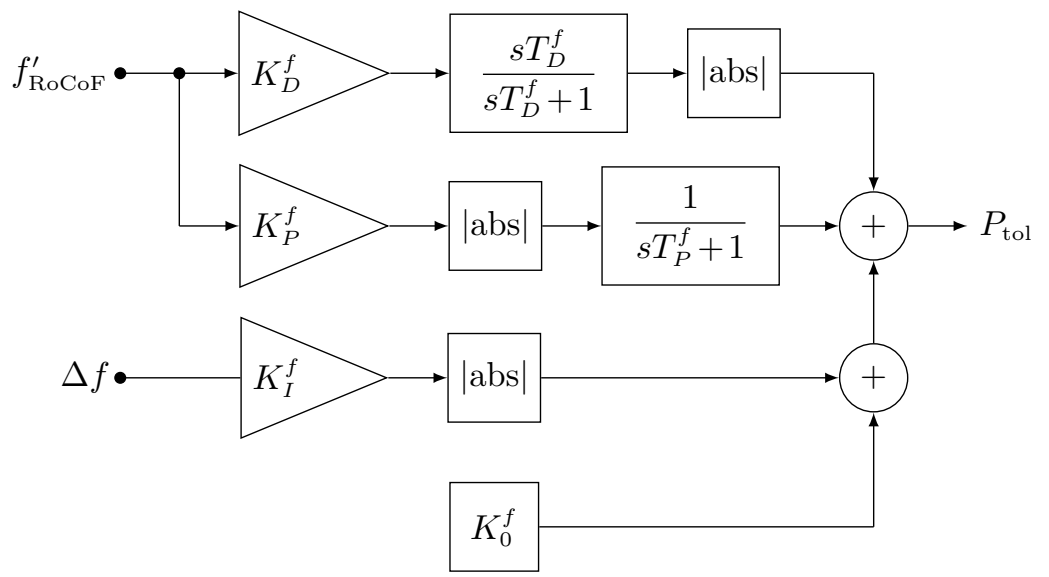

Figure 13: Calculation of the frequency support tolerance band

Table 9: Parameters of the frequency support tolerance band

\begin{tabular}{l|c|l|l} 
Parameter & Value & Unit & Explanation \\
\hline$T_{D}^{f}$ & 300 & $\mathrm{~ms}$ & derivate component time constant \\
$T_{P}^{f}$ & 40 & $\mathrm{~ms}$ & proportional component time constant \\
$K_{D}^{f}$ & 25 & - & derivative gain \\
$K_{P}^{f}$ & 1.0 & - & proportional gain \\
$K_{I}^{f}$ & 0.5 & - & integral component gain \\
$K_{0}^{f}$ & 0.02 & $\mathrm{pu}$ & base tolerance band constant
\end{tabular}

The tolerance band is continuously calculated, likewise, the differential part of the control does not have a deadband,hence, it is continuously active. However, minor response of the differential part is contained within the tolerance band. The tolerance band gets wider according to the RoCoF and the time-gradient of 
the RoCoF, i.e. second order derivative of frequency. The differential part of the tolerance band calculation creates steep surges following events of fast changing RoCoF (e.g. disturbances like disconnection of power stations or loads) as shown in Figure 14. During these steep transients, the tolerance band is widened to accommodate a practical time delay to the requirement where the behaviour has reasonable freedom in the very early moment after severe disturbances. In conclusion, the response of a WPP/WTG is considered compliant with the proposed grid code, if it remains between the tolerance bands as illustrated in Equation (3).

$$
\left|P_{\text {ref }}(t)-P(t)\right| \leq P_{\text {tol }}(t)
$$

\subsection{Frequency Response Compliance}

The control response towards the frequency test signal Figure 11 and also the compliance assessment according to the tolerance band Figure 13 are displayed in Figure 14.

The ideal PD response (Figure 12) is the blue curve. The violet curve is the response of a benchmark model of an NREL 5 MW [32] WTG that has been equipped with a pitch deloading support method by the authors. In particular, the control method proposed in [33], is adapted to achieve better alignment with the proposed generic code, mainly by tuning the deloading ratio and kinetic energy extraction droop constant. The proposed control method in Figure 12 is not integrated into the WTG model, but the benchmark support method [33] has been amended to match the Generic Grid Code requirements, such that the WTG response settles within the compliance envelope. This is to demonstrate that the grid code does not obligate a certain control method to be used, where a reasonable and fair tolerance margin, i.e. the green area between the red curves. 


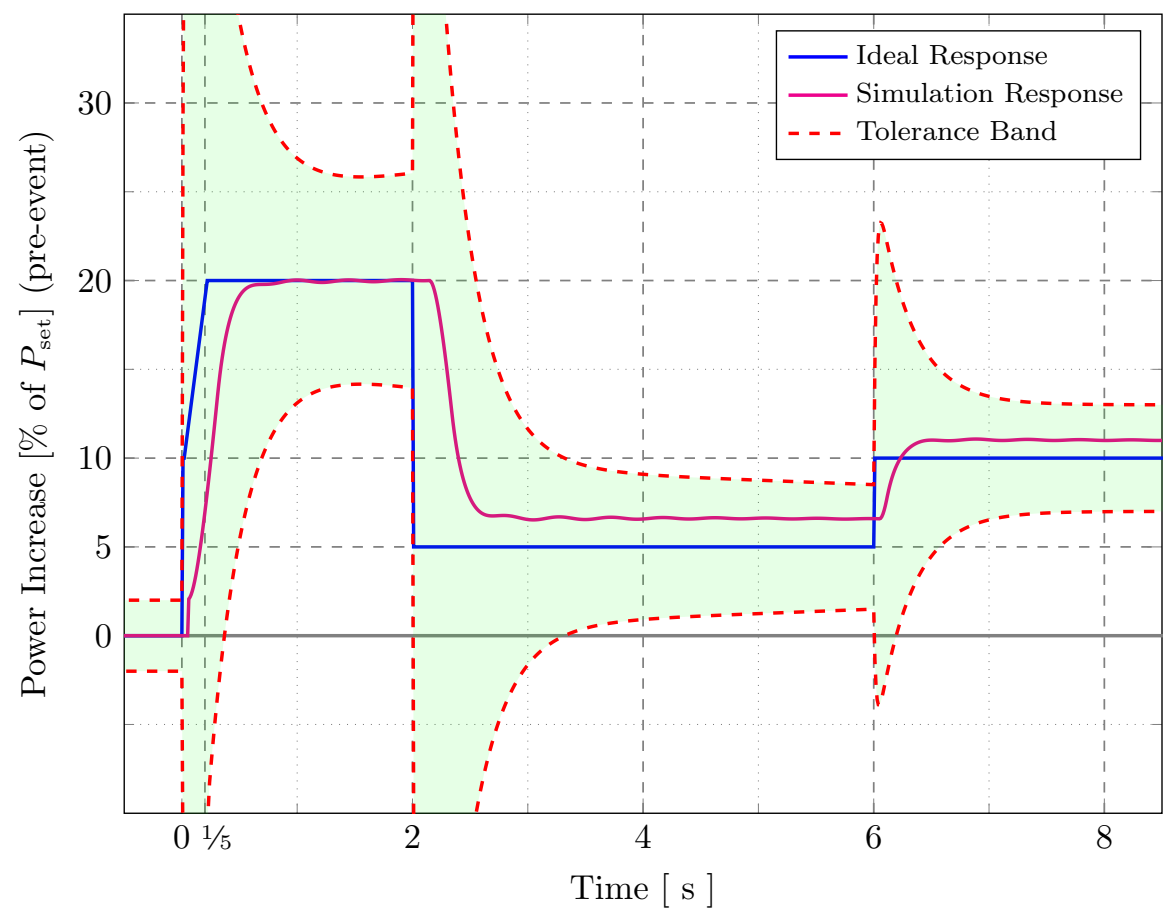

Figure 14: The power response towards the test disturbance

The green area refers to the acceptable range of responses, which includes very high ramp-up rates up to infinity. This ideal response, (i.e. infinity ramp up) to a theoretical instantaneous change in the RoCoF is an instantaneous change in power with an infinity gradient. This could be practically impossible, but a generic grid code should allow such an ideal response. The power surge magnitude $\left(P_{\mathrm{PD}}\right)$ varies between upper and lower limits, which are proposed based on the parameters' margins developed in Table 8.

The requirements of the generic grid code do require a continuous support (of the proportional control part) as long as the frequency is outside the deadband. However, it is practically challenging to maintain the output of the WPP constant within this very long time frame, due to the variations of wind speed. Hence, the proposed code response requires more suitable restriction, where the output should be kept for at least 90s inspired by some grid codes and market schemes that compare the pre-event output with the output within $90-120$ s after the event to estimate the financial compensation [34]. After this interval, violations of the requirements due to wind variations have therefore to be tolerated. Once the frequency rebounds to the deadband again, all records should be reset, and any appendage frequency event is considered as a new one, not consequential to the former event. It is worth mentioning that the proposed generic grid code prioritises voltage compliance over frequency compliance if there is no way to comply with both. This returns to two reasons, first, the voltage events are 
usually very short (the typical clearance time is around 10-15 cycles, which is around $0.3 \mathrm{~s}$ ), hence the system could recover such very short frequency events or even its natural inertia will be able to suppress it. Second, one of the voltage code requirements aspects is to maintain the active power within reasonable limits to avoid the initialization of frequency events either during the fault or after its clearance. This is also aligned with the current practices in many European grid codes.

\subsection{Considerations regarding operation at rated output}

It should be noted that the provision of proportional response component during under-frequency events (upward regulation) requires the WTGs to normally operate below maximum available power which is called deloaded operation. Otherwise, a limited overloading of the WTG should be allowed, i.e. when the WTG is already providing its rated power. The incorporated challenge is securing the required support in situations where the WPP is already providing its rated output. A supplementary controller could curtail the normal output to secure this margin, i.e. output de-loading, or apply an alternative method. In particular, there are various concepts and methods that enable wind power to provide such response, including pitch deloading and kinetic energy extraction as the most common techniques [31].

It is a question of design optimisation to decide how much of this response is coming from the WTGs, and if additional hardware like short-term energy storage is needed to comply with the grid code. A battery energy storage system (BESS) included in the WPP could supply the required frequency response and hedge forecasting errors, and provide ancillary services on behalf of the WPP as a bonus [35].

Pitch deloading could be a second choice to provide frequency support. A power-control method where the deloading ratio $\left(D_{F}\right)$ is adjusted according to wind speed condition and frequency deviation, while it has constant value at normal frequency (i.e. within the deadband applied), detailed explanation can be found in [33].

The default $D_{F}$ in this study is $15 \%$. The original control method was independent of RoCoF signal, while the adapted one adjusts the deloading ratio $\left(D_{F}\right)$ to $3.75 \%$, when the RoCoF is persistent at non-negative value, but the frequency deviation still exists. As an illustration, the applied deloading at normal frequency conditions (within deadband) is $15 \%$ of the available power. This deloading is waived gradually as the frequency drop gets worse until it reaches zero deloading when the frequency drop reaches a certain threshold $(0.2$ $\mathrm{Hz}$ in this study). However, to achieve better compliance with this generic code, the control method has been adapted, such that the $D_{F}$ is set to 0.25 of its actual value (i.e. $D_{F}=3.75 \%$ ) when $\mathrm{RoCoF}$ is persistent at non-negative value. If the drop sustains to violate the allowed deadband for more than $6 \mathrm{~s}, D_{F}$ would be set to half its nominal value (i.e. $D_{F}=7.5 \%$ ). As seen in Figure 14 (violet curve), this achieves a very good level of compliance. The slight deviation from the ideal response is normal due to the applied control method and the persistent wind speed condition, where the adjusted $D_{F}$ does not lead to the exact outcome 
of the support requirement block (Figure 12). It is worth mentioning that this response is obtained at $10 \mathrm{~m} / \mathrm{s}$ incident wind speed, which could be considered an average wind speed for many WPPs.

\subsection{Compliance of battery and PV energy systems}

The role of non-synchronous power units, e.g. PV plants and BESS, is growing as a key provider of ancillary services to the grid. The key challenge, as explained in the previous subsection is to enable these units to maintain a reasonable power reserve and integrate simple and efficient supplementary controllers to provide these services. Meanwhile, this article is focused on wind energy but it could be useful to exploit the analogy between WPPs on one hand and PV plants as well as BESS on the other hand from the viewpoint of their ability to comply with the proposed generic grid code.

Although neither the PV plants nor the BESS has natural inertia, i.e. no rotating parts but they are static power sources, the literature provides different control methods to enable them to provide synthetic inertia similar to WTGs. These systems have a common aspect which is being connected to the grid through PE either partly, like Type 3 DFIG wind turbines or completely decoupled as PV plants, BESS and Type 4 WTGs (Full rated converter with permanent magnet synchronous generator).

There is a vast number of sizing and control methods to enable BESS to provide frequency support to the grid, including the two basic services: virtual inertia and primary response. The main idea for the sizing methods is to predict the required increment power during frequency drops that should improve the grid response, and comply with the grid code applied. These predictions are usually based on historical data of the power system frequency and generation-demand [36]. The control methods are usually based on simple PI controllers where the two possible input signals are frequency deviation and/or RoCoF [28]. the output of these controllers should be the setpoints of the active power or current of the PEC such that the unit provides active power response that complies with the grid code implied. The BESS are commonly charged by the excess renewable generation (some references call it the rejected energy) during low demand intervals or during transmission systems congest ions, hence the energy of renewable units is used to charge nearby BESS [37].

Many researchers have proposed and examined the possibility of applying the mature control methods, used in WTG to provide frequency support, to PV units. As mentioned earlier, both energy systems are connected to the grid through PEC, hence the main challenge is to maintain a reasonable primary reserve and mimic synchronous generation inertia response. The well-known Perturb and Observe control method used in PV modules for Maximum Power Point Tracking (MPPT) has been modified in some studies such that the reference voltage is either shifted to the left or right of optimum tracking behaviour [38]. However, the main challenge facing this approach is being an online control method which could be slightly slower and causes unnecessary energy loss (i.e. unconverted solar irradiance to electric energy). To overcome these drawbacks, some studies proposed offline control method, which is simply a 2D lookup table receiving the 
solar irradiation and module temperature as inputs and provides the optimum operating voltage and current, which are used to adjust the setpoints of the buck-boost converter and hence the grid-side inverter [39]. To enable frequency support simple droop controller (similar to this in a synchronous generator) could manipulate the produced increment power during frequency deviations. During normal operation (i.e. when the frequency is within the safe deadband according to the implemented grid code), the PV module output is continuously deloaded to maintain a certain amount of reserve either as a fixed predetermined value (say $10 \%$ of its rated power, called Balance deloading) or fixed percentage of the available optimum output (Delta deloading).

This should provide the reader with the basic knowledge that demonstrates the ability of PV plants as well as BESS to comply with the active power requirements of the proposed generic codes. Actually, they should be more compliant, i.e. better aligned with the ideal response required by the grid code, compared to WTGs. In particular, BESS proved to be slightly faster when they provide synthetic inertia compared to WTGs in some studies [40].

\section{Conclusions}

A future-oriented generic grid code is proposed to enable the assessment of grid code compliance in a generalised and future-oriented manner, rather than examining it with respect to actual country/region-oriented grid codes of today. The generic grid code provides fault ride through voltage profile and the required response, as well as the active power response to a typical frequency event. The specifications are inspired by the ENTSO-E grid code framework and the Irish grid code, which could be considered an advanced model regarding the integration of high shares of wind energy in the generation mix.

The proposed generic grid code targets the research community and early-stage technology development. However, parts of this work could serve as inspiration for future grid code improvements, and thereby contribute to the significant efforts pushing towards large shares of renewable energy in the generation mix. In particular, European TSOs have already taken wide steps towards influential roles for wind energy in the provision of ancillary services. This naturally leads to various changes in the grid codes implemented. In addition, the proposed generic code is designed to cover most of the challenges that are not usually covered by actual codes, but at the same time, it does not follow very special protocols that adversely deviate from common TSOs practice, to maintain the balance between applicability and practical novelty. It could also contribute to bridging the gap between the lengthy and legal-blended nature documents that are published by TSOs and the researchers' and developers' objectives to have a concise reference of generic requirements.

The proposed generic grid code is based on parameterised mathematical models that could be adapted to the users' needs, e.g. addressing a small island grid. One of the key merits for this code is that it could be implemented within a short time through the presented block diagrams and equations while most grid codes are legal documents with hundreds of pages. In this context, 
the ENTSO-E RfG provides a set of boundaries to operate generation units in Europe's networks, e.g. withstand a certain frequency deviation without disconnecting. However, when addressing the response towards these deviations, ENTSO-E directs the reader to the corresponding TSO for details, which means that no generic response is included in the ENTSO-E RfG. Hence, it could be always questioned the rationale of any researchers to assess their control methods or technology developers to validate compliance of their generation units against a specific grid code.

The simulation results show that the tested wind turbine generator complies with most of the proposed requirements for both branches, i.e., voltage and frequency. However, minor deviations are observed, as the fault is cleared or when the frequency stabilises at a new value different from its nominal value. It is also promising to demonstrate the compliance with the strict requirements of reactive current, meanwhile the active current is maintained almost constant to ensure quick active power recovery after fault clearance.

\section{Acknowledgement}

The authors would like to thank Nicolaos Antonio Cutululis, Ömer Göksu, Jayachandra Sakamuri and Damian Flynn for the discussions and inputs.

\section{Bibliography}

\section{References}

[1] Til Kristian Vrana, Damian Flynn, Emilio Gomez-Lazaro, Juha Kiviluoma, Davy Marcel, Nicolaos Cutululis, and J. Charles Smith. Wind power within european grid codes: Evolution, status and outlook. Wiley WIREs Energy and Environment, 2018.

[2] EirGrid. Eirgrid grid code - version 6.0. EirGrid, 2015.

[3] M. Tsili and S. Papathanassiou. A review of grid code technical requirements for wind farms. IET Renewable power generation, 2009.

[4] Nathan Baeckeland, Bert Herteleer, and Michael Kleemann. Modelling fault behaviour of power electronic converters. International Journal of Electrical Power \& Energy Systems, 123:106230, 2020.

[5] A. Lorenzo-Bonache, A. Honrubia-Escribano, J. Fortmann, E. Artigao, and E. Gómez-Lázaro. Fault transient response of generic type 3 wind turbine models: Limitations and extension of the validation methodology. International Journal of Electrical Power \& Energy Systems, 121:106001, 2020 .

[6] C. Yongning, L. Yan, L. Zhen, C. Ziyu, and L. Hongzhi. Study on grid-connected renewable energy grid code compliance. In 2019 IEEE Sustainable Power and Energy Conference (iSPEC), pages 72-75, 2019. 
[7] Mostafa Bakkar, Santiago Bogarra, Alejandro Rolan, Felipe Córcoles, and Jaume Saura. Voltage sag influence on controlled three-phase grid-connected inverters according to the spanish grid code. IET Generation Transmission E Distribution, 14:1882-1892, 052020.

[8] Bongane Nhlapo and Kehinde Awodele. Review and comparison of the south african grid code requirements for wind generation with the european countries' grid codes. pages 1-6, 012020.

[9] Dhany Harmeidy Barus and Rinaldy Dalimi. The requirement of indonesian grid code adaptation toward variable renewable energy penetration (case study: Solar power plant in kupang sub system). Proceedings of the 2nd International Conference on High Voltage Engineering and Power Systems: Towards Sustainable and Reliable Power Delivery, ICHVEPS 2019. Institute of Electrical and Electronics Engineers Inc., oct 2019.

[10] Ruth Domínguez, Giorgia Oggioni, and Yves Smeers. Reserve procurement and flexibility services in power systems with high renewable capacity: Effects of integration on different market designs. International Journal of Electrical Power E Energy Systems, 113:1014 - 1034, 2019.

[11] ENTSO-E. Regulations - commission regulation (eu) 2016/631 - of 14 april 2016 - establishing a network code on requirements for grid connection of generators. Official Journal of the European Union, 2016.

[12] ENTSO-E. Draft network code on high voltage direct current connections and dc-connected power park modules. Official Journal of the European Union, 2014.

[13] Til Kristian Vrana, Lluis Trilla, and Ayman Attya. "development of a generic future grid code regarding wind power in europe". 16th Wind Integration Workshop, Berlin, 2017.

[14] A.B. Attya, O. Anaya-Lara, P. Ledesma, and H.G. Svendsen. Fulfilment of grid code obligations by large offshore wind farms clusters connected via hvdc corridors. Energy Procedia, 94:20 - 28, 2016. 13th Deep Sea Offshore Wind R\&D Conference, EERA DeepWind'2016.

[15] IEC 61000-4-30. Testing and measurement techniques - Power quality measurement methods. , International Electrotechnical Commission, 2003.

[16] C. L. Fortescue. Method of symmetrical co-ordinates applied to the solution of polyphase networks. Transactions of the American Institute of Electrical Engineers, 1918.

[17] D. Yazdani, M. Mojiri, A. Bakhshai, and G. Joos. A Fast and Accurate Synchronization Technique for Extraction of Symmetrical Components. IEEE Transactions on Power Electronics, 24(3):674-684, March 2009. 
[18] S. S. Yu, T. K. Chau, T. Fernando, and H. H. C. Iu. An enhanced adaptive phasor power oscillation damping approach with latency compensation for modern power systems. IEEE Transactions on Power Systems, 2018.

[19] National Grid. The Grid Code. Issue 5, Revision 4, UK, August 2013.

[20] L. Trilla, J. Pegueroles, J. Urresty, C. Muñiz, and O. Gomis-Bellmunt. Generator Short-Circuit Torque Compensation in Multichannel Wind Turbines. IEEE Transactions on Industrial Electronics, 64(11):8790-8798, Nov 2017.

[21] J. Ma, Y. Qiu, Y. Li, W. Zhang, Z. Song, and J. S. Thorp. Research on the impact of dfig virtual inertia control on power system small-signal stability considering the phase-locked loop. IEEE Transactions on Power Systems, 32(3):2094-2105, 2017.

[22] A. B. Attya and T. Hartkopf. Wind farms dispatching to manage the activation of frequency support algorithms embedded in connected wind turbines. International Journal of Electrical Power $\mathscr{E}$ Energy Systems, 53:923-936, 2013.

[23] Liu Hongzhi and Chen Zhe. Contribution of VSC-HVDC to Frequency Regulation of Power Systems With Offshore Wind Generation. IEEE Transactions on Energy Conversion, 30(3):918-926, 2015.

[24] A. Attya, O. Anaya-Lara, and W. Leithead. Novel metrics to quantify the impacts of frequency support provision methods by wind power. IEEE Innovative Smart Grid Technology, Europe, 2016.

[25] K. Narendra and T. Weekes. Phasor Measurement Unit (PMU) communication experience in a utility environment. In Cigre conference, Winnipeg, 2008.

[26] Ayman Bakry Attya and Thomas Hartkopf. Wind turbine contribution in frequency drop mitigation - modified operation and estimating released supportive energy. IET Generation, Transmission and Distribution, 8(5):862 $-872,2014$.

[27] D. Stimoniaris, D. Tsiamitros, and E. Dialynas. Improved Energy Storage Management and PV-Active Power Control Infrastructure and Strategies for Microgrids. Power Systems, IEEE Transactions on, 31(1):813-820, 2016.

[28] Ayman B. Attya, José Luis Domínguez-García, F.D. Bianchi, and Olimpo Anaya-Lara. Enhancing frequency stability by integrating non-conventional power sources through multi-terminal hvdc grid. International Journal of Electrical Power \& Energy Systems, 2018.

[29] Andrew Keane, Michael Milligan, Chris J. Dent, Bernhard Hasche, Claudine D'Annunzio, Ken Dragoon, Hannele Holttinen, Nader Samaan, Lennart Soeder, and Mark O'Malley. Capacity Value of Wind Power. IEEE Transactions on Power Systems, 26(2):564-572, 2011. 
[30] Ayman Attya and Bharath Subramanian. Impact of wind farms capacity factor and participation in frequency support - reliability analysis. IEEE 5th International Conference Power Engineering, Energy and Electrical Drives, 2015.

[31] A. B. Attya, J. L. Dominguez-Garcia, and O. Anaya-Lara. A review on frequency support provision by wind power plants: Current and future challenges. Renewable and Sustainable Energy Reviews, 2018.

[32] Aeolus FP7. Simplified NREL5MW turbine for Simulink. , Aeolus European funded FP7 project, 2012.

[33] Ayman Attya and J. L. Dominguez-García. Insights on the Provision of Frequency Support by Wind Power and the Impact on Energy Systems. IEEE Transactions on Sustainable Energy, 9(2):719-728, 2018.

[34] A. Ivanova. Frequency support with wind power participation. Master thesis, Polytechnic University of Catalonia, 2016.

[35] Chen Shuaixun, Zhang Tian, H. B. Gooi, R. D. Masiello, and W. Katzenstein. Penetration Rate and Effectiveness Studies of Aggregated BESS for Frequency Regulation. Smart Grid, IEEE Transactions on, 7(1):167-177, 2016.

[36] Mohammad Reza Aghamohammadi and Hajar Abdolahinia. A new approach for optimal sizing of battery energy storage system for primary frequency control of islanded microgrid. International Journal of Electrical Power $\&$ Energy Systems, 54:325 - 333, 2014.

[37] Mathew Aneke and Meihong Wang. Energy storage technologies and real life applications - a state of the art review. Applied Energy, 179:350 - 377, 2016.

[38] M. Killi and S. Samanta. "modified perturb and observe mppt algorithm for drift avoidance in photovoltaic systems". IEEE Transactions on Industrial Electronics, 2015.

[39] F. Jibji-Bukar and O. Anaya-Lara. Frequency support from photovoltaic power plants using offline maximum power point tracking and variable droop control. IET Renewable Power Generation, 13(13):2278-2286, 2019.

[40] P. V. Brogan, R. J. Best, D. J. Morrow, K. McKinley, and M. L. Kubik. Effect of bess response on frequency and rocof during underfrequency transients. IEEE Transactions on Power Systems, 34(1):575-583, 2019. 